\title{
Combined vascular and neurologic injury after cephalic vein cutdown approach for ICD implantation
}

\author{
Jack Xu${ }^{1}$, Peyton Card ${ }^{* 2}$, Evan Watts ${ }^{2}$, Guillermo A. Escobar ${ }^{3}$, Gareth Tobler ${ }^{4}$, Hakan Paydak \\ ${ }^{1}$ College of Medicine, University of Arkansas for Medical Sciences, Little Rock, AR, USA \\ ${ }^{2}$ Department of Internal Medicine, University of Arkansas for Medical Sciences, Little Rock, AR, USA \\ ${ }^{3}$ Division of Vascular Surgery, University of Arkansas for Medical Sciences, Little Rock, AR, USA \\ ${ }^{4}$ Division of Cardiothoracic Surgery, University of Arkansas for Medical Sciences, Little Rock, AR, USA \\ ${ }^{5}$ Division of Cardiology, University of Arkansas for Medical Sciences, Little Rock, AR, USA
}

Received: December 13, 2016

DOI: $10.5430 /$ crim.v4n1p75
Accepted: January 17, $2017 \quad$ Online Published: Februay 7, 2017

URL: https://doi.org/10.5430/crim.v4n1p75

\begin{abstract}
In this article we discuss two cases that highlight possible complications of cardiac device implantation. In particular, our first case involves a patient who, during implantable cardioverter defibrillator (ICD) implantation, sustained injuries to her subclavian artery and vein and subsequently developed a self-resolving neuropraxia of the brachial plexus. In our second case, the patient, also during ICD implantation, had his left cephalic vein nicked during cutdown. Post-op he then developed a hematoma-induced left brachial plexus injury that also eventually self-resolved. A literature search has not shown other incidences of iatrogenic brachial plexus injuries from ICD implantation as described.
\end{abstract}

Key Words: Vascular injury, Cephalic vein cutdown, Device implantation complication, Iatrogenic brachial plexopathy

\section{INTRODUCTION}

Bleeding and vascular injury are well-known peri-procedural complications of device implantation. ${ }^{[1-5]}$ Subclavian artery injury is a well known and widely reported complication of device implantation; ${ }^{[6]}$ however, combined subclavian artery and subclavian vein injury during cephalic vein cut down and pocket formation is not well reported. We report two cases detailing the mechanism of subclavian artery and vein injury during cephalic vein cut down in the first case and during blunt dissection of the pocket in the second case.

\section{CASE Descriptions}

\subsection{Case 1}

We present a 62-year-old woman with multiple sclerosis who had ischemic cardiomyopathy with a left ventricular ejection fraction of less than 35\% and New York Heart Association Class III symptoms, despite optimal guideline-directed medical therapy. The initial treatment plan was to implant a subcutaneous implantable cardioverter defibrillator (ICD). Due to her cachexia (body mass index of 17.5) and lack of fat tissue over the pectoralis muscle, it was decided to implant the single-chamber ICD under the pectoralis muscle.

An incision was made at the left deltopectoral groove and the left cephalic vein was exposed in preparation for lead placement. While using electrocautery and blunt dissection to create a pocket under the pectoralis muscle nearby the clavicle, the patient started to have overt bleeding. The apparent source of bleeding was electrocauterized to attempt hemostasis. A surgical laparotomy pad was placed in the pocket

*Correspondence: Peyton Card, MD; Email: pcard@uams.edu; Address: 4301 W. Markham St. Slot \# 634, Little Rock, AR 72205, USA. 
while cephalic vein was cannulated but could not pass a wire. However, after removal of the pad the patient continued to bleed extensively. Further attempts to achieve hemostasis with electrocautery failed. The operator attempted to surgically tie the vessels but was unsuccessful. Cardiothoracic and vascular surgery were consulted emergently, and after further surgical dissection it was discovered that the source of the severe bleeding was an injury to both the left subclavian artery and vein. Both were repaired with interposition Gore-Tex grafts. The patient required four units of blood transfusion post operatively.

Post-operative examination revealed left upper extremity weakness with diminished sensation in a C5 and C6 distribution. Neurology was consulted who determined that there was a likely thermally-induced neuropraxia of the brachial plexus. With conservative measures including physical therapy, the patient's motor function of the left upper extremity improved considerably but has not returned to baseline yet. The patient was placed on warfarin for one month to prevent early graft thrombosis of the left subclavian vein, and subsequently underwent successful and uncomplicated transvenous right-sided single chamber ICD implantation.

\subsection{Case 2}

Our second case was a 61-year-old African-American man with New York Heart Association class III systolic congestive heart failure secondary to non-ischemic, dilated cardiomyopathy. He developed a left bundle branch block with QRS duration of 161 milliseconds. The patient already had a dual chamber ICD placed previously and was deemed an appropriate candidate to upgrade his dual-chamber ICD to a biventricular ICD via the left cephalic vein. ${ }^{[7]}$ Pre-operative left upper extremity venous duplex ultrasound did not show any obstruction. During cut down, the cephalic vein was inadvertently nicked deep in the pocket close to the junction with the subclavian vein. This resulted in profuse bleeding. Hemostasis could not be achieved with conventional measures and cardiothoracic surgery was consulted emergently for assistance. The surgeon was able to tie the cephalic vein to achieve adequate hemostasis. The patient then received a left ventricular lead via the left subclavian vein and the device was upgraded to a biventricular ICD in the same session as shown in Figure 1.

Post-operatively, the patient developed left arm swelling and duplex ultrasound showed abnormal sluggish flow of the left internal jugular vein and left subclavian vein suggestive of proximal obstruction, likely the left innominate vein. Therefore, he was placed on warfarin for six months to treat the possible vein thrombosis. The left upper extremity swelling eventually resolved with time and physical therapy. He also developed a left brachial plexus injury which was thought to be caused by a hematoma as seen in Figure 2.

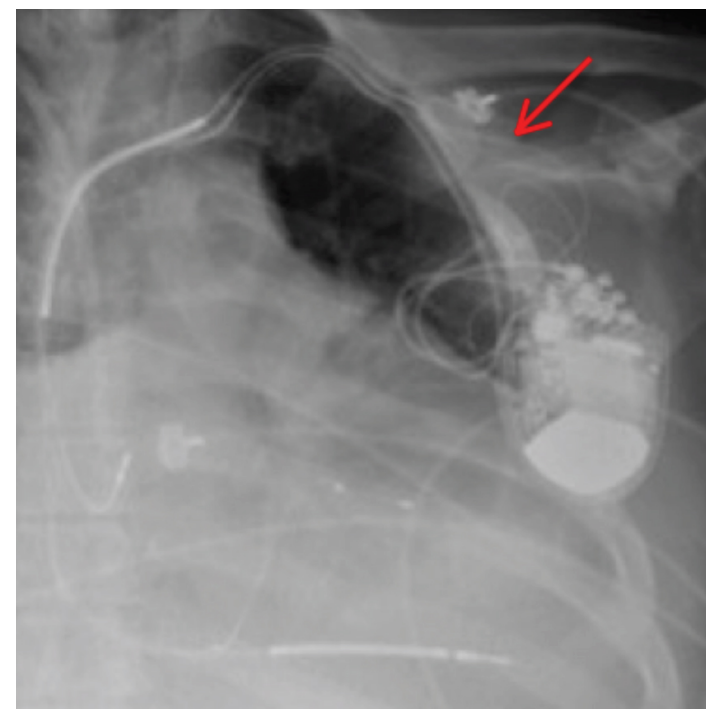

Figure 1. Posterioranterior chest radiograph showing insertion point of the new left ventricular lead through the subclavian vein via subclavicular entry. The old right atrial and right ventricular leads from the initial device placement procedure can also be seen going through the axillary vein

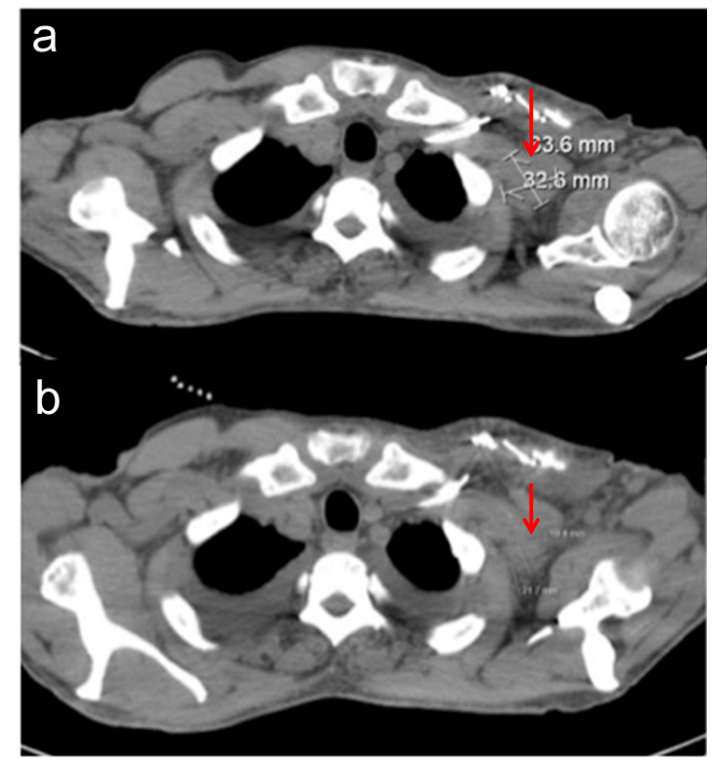

Figure 2. a. CT chest without contrast 4 months after the procedure demonstrates a soft tissue mass in the left subclavicular location in the axillary region lateral to the chest wall. This is suggestive of a hematoma along the course of the brachial plexus that is felt to have caused the patient's brachial plexopathy; b. Following CT chest without contrast 7 months post-procedure revealed that the density in the axillary region decreased in size, which is consistent with resolving hematoma 
Neurology performed an electromyography study and diagnosed the patient with a left brachial plexopathy with involvement of the upper, middle, and lower trunks. There was also a significant axonal loss in the median, ulnar, musculocutaneous and to a lesser extent radial nerves. Neurosurgery did not think that surgery would be of any benefit and fortunately the left brachial plexus function improved considerably but had not returned to baseline 14 months after the injury.

\section{Discussion}

The rate of peri-procedural complications with device implantation has been reported to be from $3 \%$ to $6 \% .{ }^{[8]} \mathrm{A}$ meta-analysis of patients undergoing device implantation who had not received any anticoagulation reported the incidence of bleeding as 2.2 out of 100 cases. $^{[9]}$ In our two cases, we report examples of rare complications of device implantation due to direct injury to subclavian vein and artery during cephalic vein cut down in the first and during subpectoralis pocket formation in the second case. Iatrogenic brachial plexus injuries from surgery and other medical procedures contribute to about 7 to 10 percent of all brachial plexopathies. ${ }^{[10,11]}$ There has been one case in which a pa- tient, after undergoing cardiac resynchronization therapy (CRT) device implantation, complained of "electric shocklike" pain in the left axillary area and was found to have brachial plexus irritation by angulated CRT leads. ${ }^{[12]}$ Classic postoperative paresis from brachial plexopathy can be caused by traction or compression during surgery. ${ }^{[10,13]}$ This typically presents as a painless weakness in the distribution of the upper brachial plexus and can also be accompanied by paresthesias. The lesions are usually a demyelinating conduction block. We believe that in our cases, the brachial plexopathy was a result from a combination of electrocautery in the first case and extrinsic compression from a hematoma in the second case. Cauterization and dissection in deep tissues immediately under the clavicle with poor visibility should be avoided at all times, and digital compression with or without topical hemostatic agents should be preferred. Having access to surgical back-up available for intra-operative support to help expose and control the source of complicated bleeding is of great value.

\section{CONFLiCTS OF INTEREST Disclosure}

The authors have no competing interests to declare.

\section{REFERENCES}

[1] Moss A, Hall W, Cannom D, et al. Cardiac-Resynchronization Therapy for the Prevention of Heart-Failure Events. N Engl J Med. 2009; 361: 1329-1338. PMid:19723701 https://doi.org/10.1056/ NEJMoa0906431

[2] Parsonnet V, Bernstein AD, Lindsay B. Pacemaker-Implantation Complication Rates: An Analysis of Some Contributing Factors. J Am Coll Cardiol. 1989; 13: 917-921. https://doi.org/10.101 6/0735-1097 (89) 90236-2

[3] Tobin K, Stewart J, Westveer D, et al. Acute Complications of Permanent Pacemaker Implantation: Their Financial Implication and Relation to Volume and Operator Experience. Am J Cardiol. 2000; 85: 774-776. https://doi.org/10.1016/S0002-9149(99) 008 61-9

[4] Darda S, Khouri Y, Gorges R, et al. Feasibility and Safety of SameDay Discharge after Implantable Cardioverter Defibrillator Placement for Primary Prevention. Pacing Clin Electrophysiol. 2013; 36: 885891. PMid:23617456 https://doi .org/10.1111/pace. 12145

[5] Van Rees JB, de Bie MK, Thijssen J, et al. Implantation-related complications of implantable cardioverter-defibrillators and cardiac resynchronization therapy devices: a systematic review of randomized clinical trials. J Am Coll Cardiol. 2011; 58: 995-1000. PMid:21867832 https://doi.org/10.1016/j.jacc.2011.06.007

[6] Williams JL, Stevenson RT. Complications of Pacemaker Implantation. Current Issues and Recent Advances in Pacemaker Therapy.
2012. https://doi.org/10.5772/48682

[7] Watts TE, Pant S, Reddy S, et al. Cephalic Vein Cutdown for Left Ventricular Lead Placement in Biventricular Device Upgrades. The Journal of Innovations in Cardiac Rhythm Management. 2015; 6: 1906-1907.

[8] Atwater BD, Daubert JP. Implantable Cardioverter Defibrillators: Risks Accompany the Life-Saving Benefits. Heart. 2012; 98 : 764 772. PMid:22422588 https://doi.org/10.1136/heartjnl-2 012-301853

[9] Bernard ML, Shotwell M, Nietert PJ, et al. Meta-Analysis of Bleeding Complications Associated with Cardiac Rhythm Device Implantation. Circ Arrhythm Electrophysiol. 2012; 5: 468-474. PMid:22534249 https://doi.org/10.1161/CIRCEP.111.969105

[10] Ferrante MA. Brachial plexopathies: classification, causes, and consequences. Muscle Nerve. 2004; 30: 547. PMid:15452843 https : //doi.org/10.1002/mus. 20131

[11] Wilbourn AJ. Iatrogenic nerve injuries. Neurol Clin. 1998; 16: 55 https://doi .org/10.1016/S0733-8619(05)70367-4

[12] Kim SY. Brachial Plexus Injury Caused by Indwelling Axillary Venous Pacing Leads. Korean Circ J. 2015; 45: 428-431. PMid:26413112 https://doi.org/10.4070/kcj.2015.45.5. 428

[13] Wilbourn AJ. Plexopathies. Neurol Clin. 2007; 25: 139. PMid:17324724 https://doi.org/10.1016/j.ncl.2006.11. 005 